\title{
Aktives Eiweiß und Tannin in Pflanzenzellen.
}

Von 0. Loew und Th. Bokorny.

In den Proceedings of the Meeting of the Koninklijke Akademie van Wetenschappen te Amsterdam vom 26. März d. J. wurde eine Abhandlung von Prof. C. van Wisselingh mitgeteilt, in welcher Koffein und Antipyrin als neue Mittel der Abscheidung von Tannin in Pflanzenzellen erklärt werden. Zugleich wurde darauf hingewiesen, daß Loew und Bokorny bereits jene beiden Basen gebraucht hätten, um das nichtorganisierte labile Protein in lebenden Zellen zur Ausscheidung $\mathrm{zu}$ bringen. Wisselingh hält die durch jene Basen erhaltene Ausscheidung lediglich für gerbsaures Koffein resp. gerbsaures Antipyrin, während wir außer allen Zweifel gestellt haben, daß die kugeligen Ausscheidungen durch jene Basen in Zellen von Spirogyren und überhaupt in äußerst zalllreichen pflanzlichen Objekten aus den vorschiedensten Familien hauptsächlich einen äußerst labilen Proteinstoff enthalten. Dieser nimmt bei seiner Ausscheidung sämtlichen Gerbstoff der Zellen mit sich. Wenn Wisselingh in seiner Abhandlung sagt l. c. S. 700: „I adhere to my opinion that antipyrine and coffeine solutions are valuable tannin reagents and suppose that Loew and Bokorny have given an inaccurate explanation of the phenomenon which they observed", so stimmt die erste Hälfte dieses Satzes mit unseren Beobachtungen überein, aber nicht die zweite.

$\mathrm{Da} B \mathrm{~W}$ isselingh meint, unsere Folgerungen seien eine ,inaccurate explanation of the phenomenon", erklärt sich wohl nur daraus, daß er unsere diesbezüglichen Studien nur sehr unvollständig kennt. Diese Studien, die sich durch fast 10 Jahre hinzogen, sind in Kap. VII und VIII der Schrift von O. Loew zusammengefaßt worden: Die chemische Energie der lebenden Zellen. In dieser Schrift ${ }^{1}$ ) findet sich bezüglich des Gerbstoffnachweises folgende Mitteilung: „Werden gerbstoffhaltige Zellen mit Koffein behandelt, so scheidet sich mit den Proteosomen zugleich auch der sämtliche Gerbstoff aus, welcher in diesen so fest gehalten wird, daß er weder mit verdünntem Ammoniak noch mit Alkohol ganz entfernt werden kann. Selbst der geringste Gerbstoffgehalt der Zellen läßt sich leicht auf die Weise erkennen, daß man zuerst Proteosomen mit Koffein

1) Zweite Auflage, pag. 98. Stuttgart 1906, Verlag von Fr. Grub. Wie im Kap. VII erwähnt, wurden sämtliche Studien über die Proteosomen von uns beiden in Gemeinschaft ausgeführt. 
erzeugt, dann die Objekte mit etwas feingepulvertem Eisenvitrol bestreut und nun langsam erwärmt bis zur Eintrocknung. Nach Wiederbefeuchten mit Wasser und einigem Stehen an der Luft werden die Proteosomen unter dem Mikroskop bei gerbstoffreichen Zellen dunkelblau erscheinen, selbst die geringsten Spuren verraten sich noch durch eine schwachbläuliche Färbung.“

Wie man zugeben wird, erhalten die Beobachtungen van Wisselingh's keineswegs eine Widerlegung unserer Auffassung, denn wir stimmen insofern mit ihm überein, daß sich Koffein und Antipyrin sehr gut zur Gerbstoffabscheidung eignen.

In unserer zitierten Schrift wird man alles finden, was auf den Gehalt der Koffeinausscheidungen nicht nur an Gerbstoff, sondern auch an Protein Bezug hat. Daß bei genauerer Prüfung eine Verwechslung von Proteosomen mit bloßem gerbsauren Koffein resp. gerbsauren Antipyrin möglich sein sollte, ist eigentlich undenkbar. Man betrachte nur einmal folgende Unterschiede:

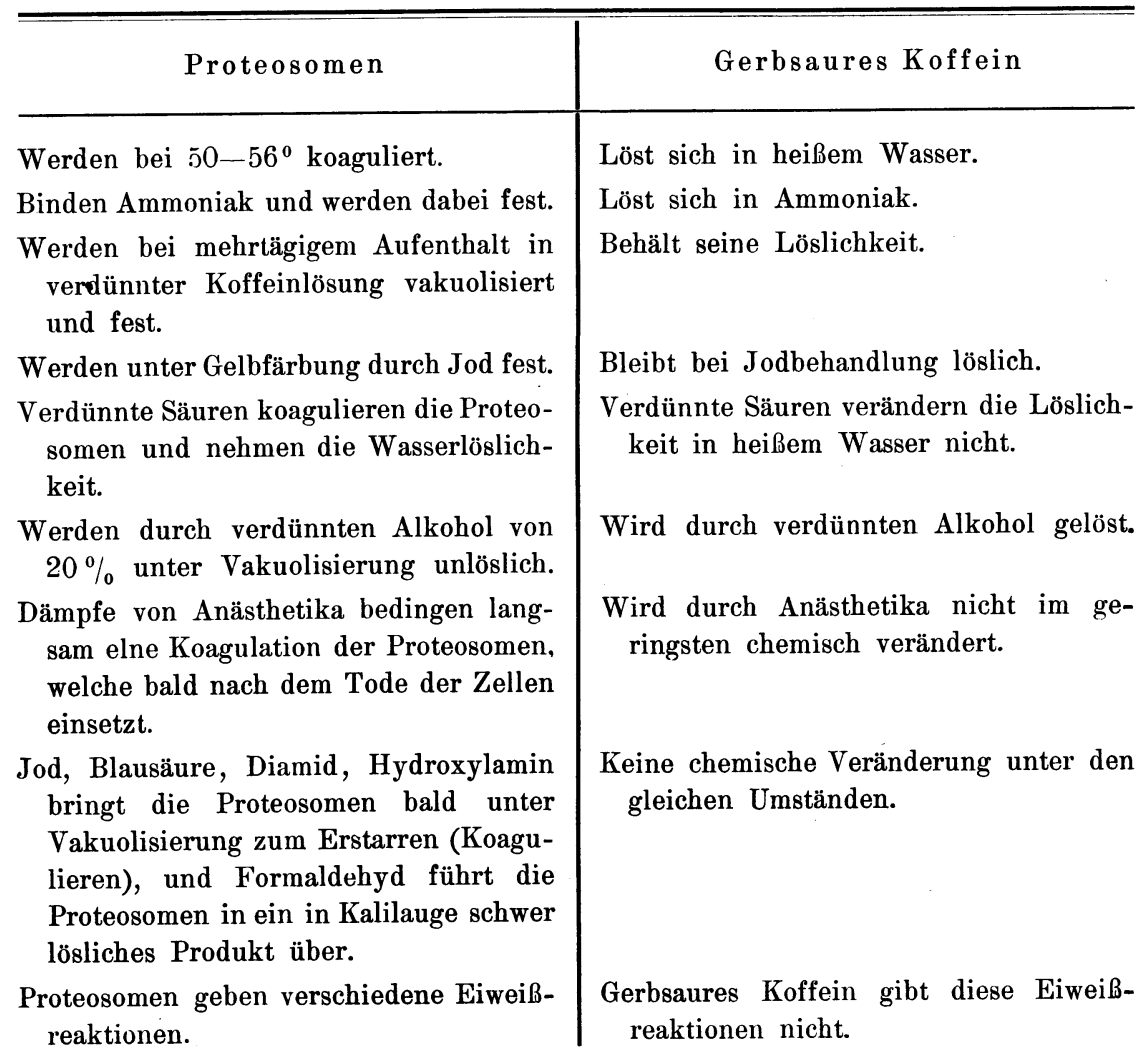


Es läßt sich zeigen, daß bei Kultur von Spirogyra in stickstofffreier Nährlösung das gespeicherte aktive Eiweiß verbraucht wird und ferner, $d a ß$ es sich wieder in den Zellen ansammelt, wenn bei Einschränkung der Zellvermehrung durch Verminderung der Phosphorsäure für die Eiweißbildung günstige Umstände hergestellt werden. Ja, diese Speicherung des labilen Eiweißkörpers läßt sich soweit treiben, daß sich derselbe in Form von Proteosomen von selbst, und ohne daß eine Spur von Koffein angewendet wird, ausscheidet ${ }^{1}$ ). Auch die spontane Ausscheidung von Eiweißkugeln beim Aushungern von Zweigen von Prunus, wobei der Ausscheidung alsbald Erhärtung folgt, sei hier kurz erwähnt.

$\mathrm{Da}$ der Gerbstoffgehalt nebensächlich ist, geht auch daraus hervor, daß gerbstoffreie Objekte, wie Schneebeeren, ebenfalls Proteosomen. liefern, ja in neuester Zeit hat Fr. Winkler ${ }^{2}$ ) beobachtet, daß auch gewisse Leukozyten proteosomenähnliche Ausscheidungen bei der Einwirkung von Koffein zeigen.

Von einigem Interesse dürfte noch sein, daß verdünnte Koffeinlösung öfters sowohl normale als anomale Plasmolyse hervorrufen kann, bei Verdünnungen, wo man diesen Effekt wahrlich nicht vermuten sollte. Merwürdig ist ferner das Verhalten von Infusorien gegenüber Koffein: unter Reizbewegungen der Tiere vergrößern sich ihre Vakuolen. Sämtliche Erscheinungen, welche das Koffein hervorruft, erklären sich am einfachsten unter dem Gesichtspunkt, daß sowohl eine Wasserausstoßung aus dem gequollenen aktiven Protein der lebenden Substanz stattfindet, als auch aus dem Bindungswasser des gelösten aktiven Albumins. Man sollte wohl vermuten, daß alle diese Verhältnisse einiges Interesse erregen könnten.

Nachschrift. Diese Einwendungen gelten auch für einen Artikel von $\mathrm{Czapek}^{3}$ ). Dieser Forscher behauptete, die Proteosomen seien in Alkohol Iöslich, was aber lediglich durch die rasche Exosmose des Koffeins vorgetäuscht wurde. In der oben zitierten Schrift, 2. Auflage, pag. 73 ist bereits darauf hingewiesen worden, daß, wenn man zunächst einen verdünnten, mit Koffein gesättigten Alkohol von 20\% 3-4 Stunden auf die Proteosomen wirken läßt, Koagulation derselben eintritt und nun starker Alkohol gar keine weitere Veränderung hervorruft.

Czapek hat Millon's- und Biuretreaktion mit den Proteosomen

1) Siehe hierüber auch Flora 1892, pag. 126.

2) Folia haematologica 1910, Bd. IX, pag. 94.

3) Berichte der Deutschen Botan. Gesellschaft, Bd. XXVIII, pag. 147. 
nicht erhalten können. Wenn er sich aber genau an unsere Vorschriften hält (l. c., pag. 74), so kann der Erfolg nicht ausbleiben.

Die wichtigsten Reaktionen für Albumine sind aber nicht jene Farbenreaktionen, sondern:

1. Koagulation durch Alkohol,

2. Koagulation durch höhere Temperatur,

3. Koagulation durch Säuren.

Wie erwähnt, reicht schon Alkohol von $20 \%$ zur Koagulation der Proteosomen aus. In siedende Kochsalzlösung getaucht, koagulieren die Proteosomen momentan; bei $56^{\circ} \mathrm{C}$ reichen ca. 5 Minuten aus (l. c., pag. 91). Säuren bringen die Proteosomen sehr bald zur Koagulation, später können die koagulierten Gebilde gelöst werden durch einen der Acidalbuminbildung ähnlichen Vorgang. Salpetersäure von $10 \%$ koaguliert die Proteosomen in 1-2 Minuten; wäscht man dann die Säure gut aus und färbt, so erhält man schöne Dauerpräparate.

Es erscheint geradezu rätselhaft, $\mathrm{da} \beta$ man dieses charakteristische Verhalten so hartnäckig ignoriert! Ist denn in der ganzen organischen Welt ein zweiter Körper bekannt, dem Koagulation nach jener dreifachen Richtung hin eigen wäre? Soll das etwa der Gerbstoff tun?

Daß aber unserem aktiven Eiweiß oder Protoprotein ein sehr labiler Zustand zukommt, geht daraus hervor, daß er zum Unterschied von Ovalbumin oder Serumalbumin

1. mit Koffein sich abscheiden läßt,

2. Ammoniak bindet, wobei ein Eiweißkörper von besonderem Verhalten resultiert,

3. durch Blausäure, Diamid und Hydroxylamin unlöslich wird,

4. einige Zeit nach dem Absterben der Zellen spontan koaguliert.

Der Umstand, daß tote Zellen keine Proteosomen mehr geben, beruht nicht auf der längst bekannten Exosmose von Gerbstoff aus toten Zellen. Wir haben diese Frage längst erledigt, aus den exosmierenden Substanzen sind keine Proteosomen mehr $\mathrm{zu}$ gewinnen ${ }^{1}$ ). Möchte man gründliche Vergleiche anstellen zwischen dem gerbsauren Koffein nach der Exosmose und wahren Proteosomen. Kugelform der kleinsten Teile bedeutet doch hier wahrlich keine Entscheidung! 2)

Wir zweifeln nicht, daß bei unparteiischer Prüfung unsere Schlüsse völlige Bestätigung finden werden.

1) l. c. pag. 91, Anm. Man versuche z. B. mit den gerbstoffreichen Galläpfeln.

2) Die Tröpfchen, welche Czapek bei Echeveria mittelst Formaldehyd erhielt, sind gewiß keine Proteosomen. Uns gelang diese Reaktion nicht. 


\section{Eingegangene Literatur.}

1) Wilh. Becker, Violae europaeae. Systematische Bearbeitung der Violen Europa's und seiner benachbarten Gebiete. Dresden-N. 1910, Verlag von C. Heinrich.

2) Beiträge zur Biologie der Pflanzen. Begründet von Fr. Cohn, herausgeg. von F. Rosen. Bd. X, Heft 1. Mit 1 Tafel. Preis: M. 5,- Breslau, J. U. Kerns Verlag. (Enthält: W. Herrmann, Über das phylogenetische Alter des mechanischen Gewebesystems bei Setaria; E. Pringsheim, Heliotr. Studien 3. Mitteilung; U. Angelstein, Über die Kohlensäureassimilation submerser Wasserpflanzen in Bikarbonat- und Karbonatlösungen; E. Pringsheim jun. und H. B. Cursky, Über Rosahefe.

3) Boletim do Museu Goeldi de historia natural e ethnographia, Vol. X. Pará 1910.

4) W. F. Bruck, Wie studiert man Biologie? Stuttgart, Verlag von Wilh. Violet. Preis: geh. M. 2,50.

5) A. Cohen-Kysper, Versuch einer mechanischen Analyse der Veränderungen vitaler Systeme. Leipzig 1910, Verlag von Georg Thieme.

6) J. M. Coulter and Ch. J. Chamberlain, Morphology of Gymnosperms with 46.2 Figures. The University of Chicago Press, Chicago. Preis: 4 Doll. 22 cts.

7) E. Döring, Das Leben der Tulpe. Mit 6 Tafeln. Sondershausen, Verlag von O. Reutter.

8) L. Fischer, Tabellen zur Bestimmung einer Auswahl von Thallophyten und Bryophyten. (Teilweise neu bearbeitet von L. Fischer.) Bern, Verlag von K. J. Wyss. Preis: M. 1.60 .

9) K. Giesenhagen, Lehrbuch der Botanik. Fünfte Auflage. Mit 557 Textfiguren. Stuttgart, Verlag von Fr. Grub. Preis: geb. M. 8,--.

10) P. Graebner, Lehrbuch der allgemeinen Pflanzengeographie nach entwicklungsgeschichtlichen und physiologisch-ökologisehen Gesichtspunkten. Mit $150 \mathrm{Ab}$ bildungen. Leipzig, Verlag von Quelle \& Meyer. Preis: geb. M. 9.-

11) G. Haberlandt, Eine botanische Tropenreise. Mit 48 Abbildungen im Text, 9 Tafeln in Autotypie und 3 Aquarelltafeln. Zweite Auflage. Leipzig, Verlag von W. Engelmann. Preis: geh. M. 11,60, geb. M. 12,85.

12) E. Jörgensen, Die Ceratien. Eine kurze Monographie der Gattung Ceratium Schranck. Mit 184 Figuren auf 10 lithographischen Tafeln. Leipzig 1911, Verlag von Dr. Werner Klinkhart. Preis: M. 7.-.

13) M. Koch, Beiträge zur Kenntnis der Höhengrenzen der Vegetation im Mittelmeergebiete. Halle a. S., Druck und Verlag von C. A. Kaemmer \& Co. Preis: M. 6,- -

14) B. Landsberg, Didaktik des botanischen Unterrichts. Leipzig und Berlin 1910, Verlag von B. G. Teubner.

15) L. Loeske, Studien zur vergleichenden Morphologie und phylogenetischen Systematik der Laubmoose. Berlin 1910, Verlag von M. Lande. 
16) Memorias do instituto Oswaldo Cruz, Tomo I, Faciculo 4. Rio de Janeiro 1909, Manguinhos.

17) A. Pascher, Chrysomonaden (Der Großteich bei Hirschberg in Nordböhmen, naturwissenschaftliche Untersuchungen veranlaßt und herausgegeben von der Gesellschaft zur Förderung der Wissenschaft, Kunst und Literatur in Böhmen). Erstes Heft des botan. Teiles. Leipzig 1910, Verlag von Dr. Werner Klinkhart. Preis: M. 10,-.

18) A. Nathanson, Tier- und Pflanzenleben des Meeres. Leipzig 1910, Verlag von Quelle \& Meyer. Preis: geh. M. 1.-, geb. M. 1,25.

19) Ders., Der Stoffwechsel der Pflanzen. Leipzig 1910, Verlag von Quelle \& Meyer. Preis :

20) R. C. Punnets, Mendelismus. Ins Deutsche übertragen von Wilfr. v. Pros kowetz. Herausgegeben, mit einem Vorwort und Anmerkungen versehen, von Dr. Hugo Iltis, Brünn. Brünn 1910, Druck und Verlag der k. u. k. Hofbuchhandlung Carl Winkler. Preis: M. 2.-.

21) G. Roth, Die außereuropäischen Laubmoose, beschrieben und gezeichnet. Erste Lieferung. Mit Tafel I-VIII. Leipzig, Verlag von C. Heinrich. Preis: M. $6,-$.

22) O. Schmeil, Lehrbuch der Botanik. Mit 40 farbigen Tafeln und zahlreichen Textbildern. Leipzig 1910, Verlag von Quelle \& Meyer. Preis: geb. M. 5,40 .

23) W. Schurig, Hydrobiologisches und Plankton-Praktikum. Eine erste Einführung in das Studium der Süßwasserorganismen. Mit einem Vorwort von R. Woltereck. Mit 215 Abbildungen im Text und 6 Tafeln. Leipzig, Verlag von Quelle \& Meyer. Preis:

24) R. Timm, Niedere Pflanzen (Naturwissenschaftl. Bibliothek für Jugend und Volk). Leipzig 1910, Verlag von Quelle \& Meyer. Preis: M. 1,80.

25) W. Wagner, Die Heide (Naturwissenschaftl. Bibliothek für Jugend und Volk). Leipzig, Verlag von Quelle \& Meyer. Preis: geb. M. 1,80.

26) R. v. Wettstein, Handbuch der systematischen Botanik. Zweite umgearbeitete Auflage, erste Hälfte. Leipzig und Wien 1910, Franz Deuticke.

27) K. Wilhelm, Die Samenpflanzen. Systematische Übersicht ihrer Familien und richtigeren Gattungen und Arten mit besonderer Berücksichtigung der für Land- und Forstwirtschaft, Technik und Arzneikunde in Betracht kommenden Gewässer. Leipzig und Wien 1910, Franz Deuticke. Preis: M. 5,- 